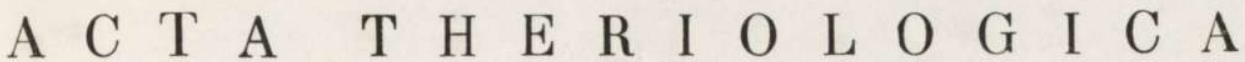 \\ VOL. XII, 17: 281-292. \\ BIAŁOWIEŻA \\ 30.VII.1967
}

Stefan HEREĆ

\section{Der Bulbus olfactorius und der Bulbus olfactorius accessorius beim Wildschwein}

[Mit Tafeln XIV u. XV]

\begin{abstract}
Den Bulbus olfactorius teilte man auf die Pars anterior und die weiter posterior von ihr liegende Pars posterior. Die Mitte des Riechkolbens nimmt der Ventriculus bulbi olfactorii ein. Die Pars anterior besteht von aussen in Richtung des Ventriculus aus folgenden Schichten: Stratum fibrosum, Stratum glomerulis olfactorii, Stratum granulosum ext. et internum und der grauen den Ventriculus umwindenden Substanz. In der Pars posterior rings um den Ventriculus ordnet sich das nasale Ende des Nucleus olfactorius ant. an. Die Nervenfasern in der Pars anterior des Riechkolbens bilden ein Nervengeflecht (Plexus nervosus), aber in der Pars posterior ordnen sich die Tractus an. Man unterscheidet den Tractus olfactorius lateralis und den Tractus olfactorius intermedius. An die dorso-mediale Seite des Riechkolbens ist der Bulbus olfactorius accessorius angeschlossen. Sein Bau erinnert an die Struktur des eigentlichen Riechkolbens.
\end{abstract}

\section{EINLEITUNG}

Mit dem Bulbus olfactorius befassten sich viele Forscher. Man untersuchte ihn bei verchiedenen Tierarten und diese Arbeiten hatten einen vielfältigen Charakter. K a ppers (1960) schreibt von seiner Struktur bei Reptilien, H u mphrey (1936) bei der Fledermaus - Tadarida mexicana, Yo ung (1936) beim Kaninchen, E us t achiewicz (1966) beim Pferd, Gadamski (1966) beim Rind. Ramon y Caja 1 (1955) untersuchte ihn bei der Maus, dem Kaninchen und der Katze. Unter anderen interessierte er sich für das Herkommen der Nervenfasern und ihren Verbindungen im Riechkolben. Johns on (1959) u. Allis on (1953) führten auf lebenden Ratten und Meerschweinchen ihre Experimente durch, um festzustellen, woher die den Tractus olfactorius bildenden Fasern herkommen. Holt (1916-17) untersuchte die Anzahl der Nervenzellen im Bulbus olfactorius und im Bulbus olfactorius accessorius bei weissen Ratten. M ann (1961) beschrieb den Bulbus clfactorius accessorius bei einigen Fledermäusen aus den Familien: Phylostomatidae und Desmodontidae. Er wies auf den Anteil dieses Gebildes beim Entstehen von sexuellen Reizen hin. 
Ausser den erwähnten Arbeiten kann man noch eine ganze Reihe von ihnen auf das Thema dieses Teiles des Riechhirns antreffen.

Als Thema der vorliegenden Arbeit wurde der Bulbus olfactorius des Wildschweines daher ausgewählt, da in der zugänglichen literatur nur die Arbeit von . $J$ a w lowski (1957) darüber berichtet, aber sie erwähnt dieses Problem nur auf eine ganz allgemeine Weise. In der „Besprechung” der vorliegenden Arbeit wurde versucht, den Bau des Riechkolbens des Wildschweines mit demjenigen des Hausschweines zu vergleichen und zwar aus Gründen der nahen Verwandschaft dieser beiden Arten. Der Bulbus olfactorius des Hausschweines wurde durch den Autor (H e r é, 1966) schon früher beschrieben.

\section{MATERIAL UND METHODE}

Die zu den Untersuchungen benutzten Riechkolben stammen von einem Eber des Wildschweines, Sus scrofa Linnaeus, 1758, der zirka 5 Jahre alt war. Vom Gehirn wurden sie vermittels eines Querschnittes in Entfernung von zirka $1 \mathrm{~cm}$ hinter ihrer posterioren Grenze abgetrennt. Weiter wurden sie 10 Tage lang in $10 \%$ und $40 \%$ Wasserlösung mit Formalin fixiert, im fliessenden Wasser gespuilt, in Äthylalkohol entwässert und in Paraffin gebettet. Der Riechkolben wurden in zwei Flächen längs und quer auf $18 \mu$ starke Schnitzel geschnitten. $\mathrm{Zu}$ Beobachtungen wurde jeder Schnitzel gebraucht. Sie wurden nach der Methode von Klüver und Earrera und nach modifizierten Methode von Landau gefärbt.

\section{EIGENE UNTERSUCHUNGEN}

1. Der Riechkolben (Bulbus olfactorius)

Im Riechkolben unterscheidet man makroskopisch zwei antero-posterior gehende Teile: Pars olfactoria anterior und Pars posterior. Der erste Teil ist von aussen mit Fila olfactoria bedeckt, die zum Riechkolben aus der Pars anterior der Nasenhöhle gelangen. Man unterscheidet in ihr die nasal gerichtete Fläche, die am grössten ist und eine antero-posterior gerichtete Stellung hat, weiter die laterale, die ventrale und mediale Fläche. Die posteriore Grenze der Pars anterior macht Fissura circularis aus, die die Pars anterior von der Pars posterior trennt. Im Innern der Pars anterior und der Pars posterior befindet sich der Ventriculus bulbi olfactorii.

Pars anterior besitzt ein vielfaches Stratum und die einzelnen Schichten legen sich konzentrisch rings um den Ventriculus bulbi olfactorii an. Von aussen in der Richtung der Kammer unterscheidet man folgende Schichten:

1. Das Stratum fibrosum wird durch Riechfäden gebildet, die zur Oberfläche des Bulbus olfactorius gelangen. Nach Erreichung dieser Oberfläche verzweigen sie sich, verflechten sich dann und dringen in die Glomeruli ein (Abb. 1). Die Riechfäden sind von verschiedener Dicke. Allgemein wird festgestellt, dass es dickere Fäden meistenteils in nasa- 
ler Richtung und dünnere in medialer gibt. Auf der nasalen Fläche gibt es ausserdem mehr Riechfäden im Vergleich mit übrigen Flächen des Riechkolbens. Die genaue Reichweite vom Stratum fibrosum in kaudaler Richtung lässt sich auf den Querschnitten feststellen. Auf ihnen stellt man fest, dass es zuerst auf der dorsalen Seite des Riechkolbens, dann auf der lateralen und ventralen endet, aber sich am längsten in kaudaler Richtung auf der medialen Seite hält. Vermittels der Methode von Klüver und Barrera färben sich die Fäden sehr schwach, denn sie sind von Markhüllę entblösst.

2. Das Stratum glomerulis olfactorii verbreitet sich im Bereich des Bulbus olfactorius genau so wie die Riechfäden. Die Fäden sind Axonen der Nervenzellen, die in der Pars anterior der Nasenhöhle liegen. In den Glomeruli besitzen sie Synapsen mit den Dendriten der mitralen und schopfartigen Zellen, die schon in den tieferen Schichten des Riechkolbens auftreten.

Die Glomeruli im Riechkolben des Wildschweines stellen meistenteils Gebilde von birnenförmiger und ovaler Gestalt dar. Runde trifft man sehr selten an (Abb. 1, 2). Alle liegen lose nebeneinander an. In der Regel überschreitet die Dicke des beschriebenen Stratum die Stärke eines Glomeruli nicht. Nur an seltenen Stellen lagern sie sich in zwei Schichten. Die Mehrzahl dieser Gebilde liegt auf einem Niveau, indem sie auf den Querschnitten einen geschlossenen Ring rings um den Riechkolben bilden. Einige lagern jedoch oberhalb des allgemeinen Niveau's, aber sehr selten unterhalb. Im Bereich der Glomeruli trifft man auf dünn gelagerte kleine oder mittlere, runde oder ovale körnige Zellen. Dieselben Zellen füllen den Raum zwischen den Glomeruli auf. Unter den Glome-ruli lagern sie als dünne wellenförmige Schicht (Abb. 2).

3. Das Stratum granulosum externum teilt sich auf drei Teile: den externen, medialen und internen. Am dicksten ist der mediale Teil, die beiden übrigen sind sehr dunn. Der externe Teil besteht aus körnigen Zellen und ist bei Stratum glomerulis olfactorii beschrieben worden.

Der mediale Teil wird auch Stratum moleculare genannt (Abb. 1). Seine graue Substanz besteht aus körnigen, mitralen und schopfartigen Zellen. Alle drei Zellenarten sind dünn zerstreut. Die körnigen Zellen haben entweder eine runde oder ovale Gestalt von kleiner und mittlerer Grösse. Zwischen ihnen treten mitrale Zellen auf. Sie sind viereckig, aber zuweilen leicht verlängert. Sie besitzen lange Fortsätze, die fast immer nach der Seite der Glomeruli gerichtet sind, aber deutliche Kerne haben. Sie färben sich gut, jedoch etwas schwächer als die eigentlichen mitralen Zellen, die weiter unten beschreiben werden. Die mitralen Zellen treten im ganzen Stratum moleculare auf. In Richtung der Riechkolbenfläche überschreiten sie diese und manchmal trifft man sie zwi- 
schen den Glomeruli an. Der letzte Zellentyp d.i. die schopfartigen Zellen haben einen spindelförmigen oder dreieckigen Umriss, ovale Kerne und eine ungrosse Menge an Zytoplasma. Ausser Zellen trifft man im Stratum moleculare schwach gefärbte, dünne Nervenfasern an. Sie verlaufen zwischen den Zellgebilden. Unter anderen sind es Dendriten der mitralen und schopfartigen Zellen, die sich zu den Glomeruli begeben.

Von der Seite des Ventriculus bulbi olfactorii zum Stratum moleculare liegt eine Schicht von typischen mitralen Zellen an (Abb. 1, 3). Dies ist der dritte, tiefste Teil von Stratum granulosum externum. Diese Zellen sind gross, von viereckiger oder dreieckiger Gestalt. Sie bilden eine einzelne Schicht und sind nebeneinander lose gelagert. Innerhalb enthalten sie deutliche Kerne, Protoplasma und feinkörniges Tigroid. In den Zwischenräumen befinden sich zwischen ihnen körnige Zellen von runder oder ovaler Gestalt. Typische mitrale Zellen im Unterschied von mitralen, die man im Stratum moleculare antrifft, besitzen deutlichere Umrisse, sind vielmehr grösser von ihnen und stärker gefärbt. Die mitralen Zellen, die sich im Straten moleculare befinden, sind dorthin aus dem Stratum der eigentlichen mitralen Zellen eingewandert.

4. Das Stratum granulosum internum nimmt den Innenteil des Riechkolbens ein. Dieses Stratum ist das dickste von allen Schichten (Abb. 1, 4). Vom Ventriculus bulbi olfactorii wird es nur durch die graue, sie umgebende, ventrikulare Substanz abgeteilt.

Der äusserliche Teil des Stratum granulosum internum ist hauptsächlich aus grossen und mittleren körnigen Zellen aufgebaut. Kleine werden selten angetroffen. Die körnigen Zellen sind rings um den Ventriculus (Bulbi olfactorii) als wenn in Ringen gelagert, die durch Streifen von Nervenfasern unterbrochen werden. Dieses Bild wird auch als inselartige Anordnung benannt, aber mit dem Vermerk, dass diese Inselchen sich konzentrisch und wechselständig anordnen.

Im ventriculär anliegenden Teil des beschriebenen Stratum überwiegen entschieden die Nervenfasern und die gesamte Zellanzahl verringert sich. In Hinsicht auf die Grösse treten hier grosse und kleine körnige Zellen auf.

Nervenfasern, die im ganzen Stratum granulosum internum auftreten, sind gut gefärbt und laufen in allen Richtungen.

5. Die graue sich um den Ventriculus windende Substanz und der Ventriculus olfactorius. Die graue um den Ventriculus von innen lagernde Substanz besteht aus grossen, mittleren und runden Zellen, die den körnigen Zellen ähneln. Zwischen den Zellen trifft man auf feine Nervenfasern. Die graue Substanz tritt im Bereich des Bulbus olfactorius wie auch der Pars posterior auf, aber in Pars posterior ist sie dicker und deutlicher. Sie wird deshalb erwähnt, weil sie sich deutlich vom Stratum 
granulosum internum unterscheidet. Von der Seite des Ventriculus ist die graue Substanz mit ependymalen Zellen ausgepolstert, die an sie eng anliegen. Der Bulbus olfactorius hat auf den Querschnitten im anterioren Teil des Kolbens die Gestalt eines Dreiecks, näher zu Pars posterior ist er dem Rechteck ähnlich, dessen längere Seite im Niveau liegt. In der Pars posterior verengt sich der Ventriculus und flacht sich dorso-ventral ab.

2. Der Bulbus olfactorius accessorius. Dieses Gebilde liegt beim Wildschwein auf der dorso-medialen Seite der Pars posterior gleich hinter der Pars anterior. Mit seinem nasalen Ende lagert er in einer Vertiefung, die im dorsalen Teil des Tractus olfactorius lateralis gebildet wird. Der Bulbus olfactorius acc. ist dorso-ventral abgeflacht. Im nasalen Teil ist er dicker, aber posterior wird er immer dünner (Abb. 5, 7). Von der dorsalen Seite ist er fast ganz durch das Neopalium zugedeckt, jedoch mit Ausnahme eines kleinen nasalen Endes. Sichtbar ist es erst bei mikroskopischen Untersuchungen. In Hinsicht auf seinen Bau unterscheiden wir in ihm ganz dieselben Schichten wie im Riechkolben, aber mit Ausnahme der grauen, um den Ventriculus lagernden Substanz und des Ventriculus olfactorius. Im Bulbus olfactorius accessorius fehlt es ebenfalls an konzentrischer Anordnung der einzelnen Schichten. Die breiteste Schicht ist das Stratum glomerulis olfactorii. Seine Dicke beträgt einige Glomeruli, die hier kleiner sind als im eigentlichen Riechkolben. Das Stratum fibrosum bildet den verzweigten $N$. vomeronasalis, der bekanntlich den Bulbus olfactorius accessorius mit dem Jacobson'schen Organ verbindet. Zwischen den Glomeruli und manchmal in ihnen selbst treten körnige Zellen auf, die für das Stratum granulosum externum typisch sind. Das Stratum moleculare ist sehr schmal und es wurden in ihm keine schopfartige Zellen festgestellt. Unter ihm lagert eine dicke Schicht von mitralen Zellen. Diese mit den mitralen Zellen im Riechkolben vergleichend, stellt man fest, dass sie von ihnen kleiner, schwächer gefärbt sind und keine deutlichen Fortsätze haben. Ihre Gestalt ist oval. Unter den mitralen Zellen liegen lose zerstreute körnige Zellen, die mit Nervenfasern verflochten sind. Diese Schicht kann man als gleichartig mit dem Stratum granulosum externum im Bulbus olfactorius halten. Unter diesen beschriebenen Elementen, auf der Höhe des nasalen Endes des Bulbus olfactorius accessorius befinden sich einige Bündel von Nervenfasern, die sich mit den Fasern des Bulbus olfactorius vermischen.

3. Der Nucleus olfactorius anterior tritt in der Pars posterior auf (Abb. $6,8,9)$. Er geht als wenn in die Pars posterior von kaudaler Seite hinein und begibt sich zur Pars anterior. Im posterioren Teil der Pars posterior umgibt der Kern den Ventriculus olfactorius rings herum und man kann hier in ihm hinsichtlich der Topographie vier Teile unterscheiden: den 
dorsalen, lateralen, ventralen und medialen. Am meisten nach vorn ist der dorsale Teil vorgeschoben, aber besonders mit seiner medialen Seite, weiter der laterale, und ventrale, aber am kürzesten ist der ventrale. Der vordere Teil des Nucleus olfactorius anterior, der in dieser Arbeit beschrieben worden ist, erinnert an ein rechteckigen Dreieck, das rings um den Ventriculus olfactorius auf solch eine Weise umwunden ist, dass die Basis die Pars posterior in ihrem kaudalen Ende umgibt, aber der Gipfel in dem am meisten vorgeschobenen Punkt des dorsalen Teiles des Kernes liegt. Der dorsale Teil des Kernes geht auf der Höhe des Neopalium in seine graue Substanz über (Abb. 6). Die Querschnitte beobachtend, stellt man fest, dass der dorsale und laterale Teil keine geraden Linien bilden, sondern sich wellenartig anordnen und dies besonders im posterioren Teil der Pars posterior. Der ventrale und mediale Teil sind kurz und ordnen sich fast in geraden Linien an.

Über dem dorsalen Teil des Kernes aber mehr anterior und mehr posterior über dem lateralen liegt ein Zellstreifen an und zwar mit denselben Zellen, wie die Kernzellen. Dieser Streifen teilt sich deutlich von der Hauptmasse des Kernes ab und ist als Pars externa des Kernes benannt worden.

4. Die weisse Substanz des Riechkolbens. Die Nervenfasern im Bulbus olfactorius treten so in seiner Pars anterior wie auch in seiner Pars posterior auf. In der Pars anterior befinden sie sich in allen Schichten, aber ihre grösste Anzahl verweilt im Stratum granulosum externum (Abb. 1). Im äusseren Teil des Stratum granulosum internum verlaufen die Fasern zwischen den Inselchen der körnigen Zellen und zwar vereinzelt oder in kleinen Bündeln. Im ventrikularen Teil dieser Schicht verringert sich die Anzahl der Nervenzellen, aber diejenige der Nervenfasern steigt an.

Auf den nach Methode Klüver und Barrera gefärbten Querschnitten lassen sich in Hinsicht der Intensität des Färbens hellere und dunklere Fasern unterscheiden (Abb. 11). Beide Arten dieser Fasern treten rings um den Ventriculus olfactorius auf. Auf den ventralen und auf den lateralen Seiten des Ventriculus ordnen sie sich in Bündeln an (im Verhältnis zum Ventriculus sind sie schräg, nasalo-inferior gerichtet) und sammeln sich in eine dicke Schicht über ihm an. Zwischen den Bündeln der dunklen Fasern verlaufen helle Fasern dicht. Sie haben einen weniger regulären Verlauf als die dunklen Fasern und bilden ebenfalls eine dicke Schicht auf der dorsalen Seite des Ventriculus, jedoch unter den dunklen Fasern. In der Pars anterior des Riechkolbens auf der ventralen und medialen Seite gibt es die meisten Fasern.

Die oben beschriebenen Fasern im Bereich der Pars anterior wurden Nervengeflecht benannt. In der Pars anterior des Riechkolbens liegt die Schicht der dunklen Fasern auf der Schicht der hellen, aber in der Pars 
posterior werden sie anfänglich durch den keilartig eindringenden dorsalen Teil des Nucleus olfactorius ant. und später hin durch die übrigen Teile dieses Kernes getrennt. Diese Teilung ist dann gänzlich, wenn der Kern ringsherum den Ventriculus olfactorius umgibt. Die über dem $\mathrm{Nu}$ cleus olfactorius liegenden Fasern bilden im Bereich der Pars posterior den Tractus olfactorius lat. Die zwischen dem Kern und dem Ventriculus liegenden Fasern gehen in den Tractus olfactorius intermedius über.

Der Tractus olfactorius lateralis ordnet sich auf der äusseren Fläche des Nucleus olfactorius ant. an (Abb. 7, 9, 12). Seine Fasern unterscheiden sich im Nervengeflecht deutlich durch eine dunklere Färbung. Am meisten nach vorn vorgeschoben ist sein dorsaler Teil, der über dem dorsalen Teil des Nucleus olfactorius ant. liegt. In einer auf der medialen Seite des dorsalen Teiles liegenden Vertiefung, die weniger reich an Fasern ist als die laterale, ist das nasale Ende des Bulbus olfactorius accessorius gelegen. Fasern unter ihr ordnen sich deutlich in runde Bündel an. Wahrscheinlich an dieser Stelle nimmt der Tractus olfactorius lat. Fasern des Bulbus olfactorius accessorius auf. Posterior wird der dorsale Teil des Tractus olfactorius lat. immer dünner. Hinter dem Riechkolben verbleibt von ihm nur eine dünne Fasernlamelle, die auf der dorsalen Seite des Bulbus olfactorius und zwar ganz auf seinem Rand liegt. Wahrscheinlich verschiebt sich der dorsale Teil auf die laterale Seite des Riechkolbens und tritt in den Bestand des lateralen Teiles des Tractus olfactorius lat. über. Der laterale Teil ist am dicksten und enthält die meisten Fasern. In der oberen Hälfte ist er dünner, dagegen in der unteren beträchtlich breiter. Posterior verschwindet er stufenweise von oben anfangend, aber mit seinem unteren Teil verschiebt er sich anfänglich auf die laterale Seite des Riechkolbens und später auf seine mediale Seite. Der mediale Teil ist sehr klein. Sehr oft haben die Zellen des anterioren Nucleus olfactorius, die in der Nähe des lateralen Tractus olfactorius liegen, Fortsätze, die sich in der Richtung des Tractus lenken. Man kann vermuten, dass die Fasern dieser Zellen in den Bestand des lateralen Tractus treten. Dieser Tractus führt ebenfalls Fasern aus dem Nervengeflecht mit sich.

Der Tractus olfactorius intermed. ist aus sich hell färbenden Fasern gebildet. Er ordnet sich auf der inneren Fläche des Nucleus olfactorius, zwischen dem Kern und dem Ventriculus an (Abb. 8). Er besteht aus einer dicken Fasernschicht, die fast parallel zum Ventriculus laufen. Vor dem Bulbus olfactorius accessorius befindet er sich nur auf der dorsalen Seite des Ventriculus. Auf der Höhe des Nebenbulbus tritt er ebenfalls auf der lateralen Seite des Ventriculus olfactorius auf. Hinter dem Bulbus olfactorius accessorius wird der Teil, der über dem Ventriculus liegt, immer dünner, denn es verringern sich die Fasern. Wahrschein- 
lich treten sie von der dorsalen Seite auf die laterale des Ventriculus aber. Auf den letzten Querschnitten ist dieser Tractus auf der lateralen Seite des Ventriculus am dicksten, aber der dorsale und laterale Teil sind verhältnismässig klein. In den Bestand des medialen Tractus olfactorius treten Fasern aus dem Nervengeflecht und wahrscheinlich aus dem anterioren Nucleus olfactorius und zwar aus demjenigen Teil, dessen Zellen ihre Fortsätze nach dem Ventriculus olfactorius richten.

\section{BESPRECHUNG}

Der Bau des Riechkolbens beim Wildschwein ist grundsätzlich mit demjenigen anderer Tiere ähnlich.

Das Stratum glomerulis olfactorii im Riechkolben des Wildschweines ist in der Regel einfach, obwohl die Glomeruli auf verschiedenen Höhen liegen, ähnlich wie beim Kaninchen (Young, 1936). Stellenweise beträgt seine Dicke zwei oder drei Glomeruli. Die Glomeruli im Riechkolben des Wildschweines sind grösser und mehr oval als beim Hausschwein (H e r e ć, 1966). Beim Hausschwein ist auch das Stratum zweifach oder dreifach, aber an derartigen Stellen gibt es mehr als beim Wildschwein. Die Glomeruli im Riechkolben des Pferdes ordnen sich meistenteils in eine zweifache Schicht an (E u s t a chi ew i c z, 1966); beim Rind bilden sich auch stellenweise zweifache oder dreifache Reihen (G a d a mski, 1966). Das Stratum granulosum ext. und Stratum granulosum int. sind wie bei anderen Tieren ähnlich. Auf Unterschiede traf man bisher nur bei den Reptilien. Bei ihnen fehlt es nach K a p pers et al. (1960) im Stratum moleculare an schopfartigen Zellen. Das Stratum granulosum int., aber besonders seinen paraventricularen Teil, beim Wild- und Hausschwein vergleichend, lässt es sich bemerken, dass es beim Wildschwein an Nervenfassern reicher ist. Die paraventriculare Substanz im Bulbus olfactorius des Wildschweines bildet eine sehr dünne Schicht. Beim Hausschwein ist sie viel dicker. Bei anderen Tieren tritt sie ebenfalls auf und ähnelt vielmehr mit der grauen, paraventricularen Substanz des Hausschweines. Der Ventriculus olfactorius ist beim Wildschwein gross, ähnlich wie beim Hausschwein (H e r eć, 1965), Pferd (E us tachewic z, 1966), Rind (G a d a mski, 1966), Kaninchen (Y o ung, 1936), bei der Ratte (A 11 is on, 1953). Bei Tadarida mexicana (H u m p re y, 1936) tritt der Ventriculus überhaupt nicht auf, aber man kann ab und zu nur das ependymale Polster jedoch ohne Licht antreffen. Der Nucleus olfactorius anterior ist beim Wildschwein sehr gut entwickelt und verhältnismässig grösser als beim Hausschwein (H e r e ć, 1966). Bei den übrigen untersuchten Tieren ist er ebenfalls gut ausgebildet. Rings um die Hauptmasse des Kernes gibt es beim Wildschwein viel vereinzelte 
Zellen, die lose angeordnet sind. Beim Hausschwein treten sie weniger zahlreich auf.

Sehr deutlich ist beim Wildschwein im Riechkolben die Pars externa. Sie tritt ebenfalls beim Hausschwein, Pferd, Rind und Kaninchen auf. Beim Rind benennt sie G a d a m ski (1966) als ,,externe Anhäufung des Kernes”, aber E us ta ch i ewic z (1966) beim Pferd als „Lamelle”. Die Nervenfasern in der Pars anterior des Riechkolbens beim Wildschwein bilden genau so wie auch bei anderen Tieren das Nervengeflecht. Dieses Geflecht zu untersuchen ist schwer. Solche Autoren wie $\mathrm{R}$ a mon y C a ja l (1955), A ll is on (1953), J o h n s on (1959) geben an, dass in diesem Geflecht ausser den abführenden Fasern auch zuführende auftreten, die aus den ausserriechkolbigen Zentren des Rhinencephalons stammen. R a m on y Ca ja l (1955) untersuchte die zentrifugalen $\mathrm{Fa}-$ sern bei der Katze, dem Kaninchen und der Maus. Seiner Meinung nach gelangen diese bis zum Stratum granulosum int., wo sie sich verzweigen, aber niemals die Schicht der mitralen Zellen überschreiten. Die Nervenfasern in den Tractus olfactorius beim Kaninchen, bei der Ratte und dem Meerschweinchen untersuchend, stellten Allison (1953) und J o hns on (1959) fest, dass der Tractus olfactorius lat. bei diesen Tieren aus Axonen der mitralen Zellen des Bulbus olfactorius, des Bulbus olfactorius accessorius und aus vom Nucleus olfactorius ant. - abgehenden Fasern besteht. Der Tractus olfactorius intermed. entsteht aus Axonen der schopfartigen Zellen, aus vom Nucleus olfactorius ant. abgehenden Fasern und aus zentrifugalen Fasern. Da der Riechkolben des Wildschweines mit denjenigen von anderen untersuchten Tieren in Hinsicht auf Zyto- und Fibroarchitektonik ähnlich ist, kann man vermuten, dass die Hauptmasse der Fasern in den Riechbahnen des Wildschweines dieselbe Abstammung hat wie bei der Ratte, der Maus oder dem Meerschweinchen. An den Riechkolben des Wildschweines ist der Bulbus olfactorius accessorius befestigt. Er tritt ebenfalls beim Hausschwein (H e r éc, 1966), Rind (G a d a m ski, 1966), Pferd (E u s ta chi ewi cz, 1966), Kaninchen (Young, 1936), bei der Ratte (A 11 is on, 1953) und dem Maulwurf (Johnson, 1957) auf. Er fehlt bei einigen Arten der Fledermäuse (H u mphrey, 1936). Nach Jaw łowski (1957) fehlt er wahrscheinlich bei dem Wiesel, Baummarder und Iltis.

Der Bulbus olfactorius accessorius beim Wildschwein ist etwas grösser als beim Hausschwein. Ausserdem ist er mehr unter das Neopalium eingedrängt. Alle Schichten dieses Nebenbulbus olfactorius beim Wildschwein sind ähnlich wie bei dem Hausschwein gut entwickelt. Bei dem Maulwurf stellte Johnson (1957) im Bulbus olfactorius accessorius keine Anwesenheit von mitralen Zellen fest. 


\section{SCHRIFTTUM}

1. All is on A. C., 1953: The structure of the olfactory bulb and its relationship to the olfactory pathways in the rabbit and the rat. J. comp. Neurol., 98: 309 -355 .

2. Eustachiewicz R., 1966: Budowa opuszki węchowej (bulbus olfactorius) konia. Annls Univ. M. Curie-Skłodowska, DD 20: 93-107.

3. G a d a mski R., 1966: Cyto- i fibroarchitektonika opuszki węchowej krowy. Annls Univ. M. Curie-Skłodowska, DD 20: 109-124.

4. Hereć S., 1966: Budowa opuszki węchowej (bulbus olfactorius) u świni. Annls Univ. M. Curie-Skłodowska, DD 20: 125-138.

5. Holt C. M., 1916/17: Part II. On the number of nerve cells in large and small olfactory bulbs. J. comp. Neurol., 27: 235-252.

6. Humphrey T., 1936: The telencephalon of the bat. I. The non-cortical nuclear masses and ceratin pertinent fiber connections. J. comp. Neurol., 65: $604-653$.

7. Jawlowski H., 1957: On the bulbus olfactorius and bulbus olfactorius accessorius of some mammals. Annls M. Curie-Skłodowska, C 10: 67-86.

8. Johns on T. N., 1957: The olfactory centers and connections in the cerebral hemisphere of the mole (Scalopus aquaticus machrinus). J. comp. Neurol., 107: $379-409$.

9. John s on T. N., 1959: Studies of the brain of the guinea pig. II. The alfactory tract and fornix. J. comp. Neurol., 112: 148-196.

10. $\mathrm{Kr}$ einer J., 1949: Myeloarchitectonics of the lateral olfactory tract and of piriform cortex of the albino rat. J. comp. Neurol., 91: 103-127.

11. Kappers A., Crosby E. C. \& H uber C., 1960: The comparative anatomy of the nervous system of vertebrates including man. H. P. C., New York.

12. M a n n G., 1961: Bulbus olfactorius accessorius in Chiroptera. J. comp. Neurol., 116: 135-141.

13. Ramon y Cajal R., 1955: Histologie du systeme nervoux de l'Homme et des Vertebres. Madrid.

14. Young M. W., 1936: The nuclear pattern and fiber connections of the non-cortical centers of the telencephalon of the rabbit (Lepus cuniculus). J. comp. Neurol., 65: 296-345.

Eingang des Ms., 15. Februar 1967.

Department of Animal Anatomy,

College of Agriculture,

Lublin, Akademicka 11, Poland.

Stefan HEREĆ

OPUSZKA WĘCHOWA I OPUSZKA WĘCHOWA DODATKOWA U DZIKA

\section{Streszczenie}

W pracy opisano budowę opuszki węchowej (bulbus olfactorius) u dzika, Sus scrofa Linnaeus, 1758. Od mózgowia opuszki odcięto $\mathrm{w}$ odległości około $1 \mathrm{~cm}$ poza ich tylną granicą. Odwadniano je w ciągu alkoholu etylowego i zatapiano w 
parafinie. Skrawki do obserwacji (18 mikronowe) barwiono według metody Klüvera i Barrery oraz według zmodyfikowanej metody Landau.

Opuszkę podzielono na część węchowa i tylnie leżącą od niej szypułę. Srodek opuszki zajmuje ventriculus bulbi olfactorii. Czẹść węchowa od zewnątrz w kierunku komory składa się z następujących warstw: włóknistej, kłębków węchowych, ziarnistej zewnętrznej, ziarnistej wewnętrznej i istoty szarej okołokomorowej. W szypule opuszki, wokół komory układa się donosowy koniec nucleus olfactorius anterior.

Włókna nerwowe w części węchowej opuszki tworzą splot nerwowy (plexus nervosus), a w szypule opuszki układają się w szlaki. Wyróżniono tractus olfactorius lateralis i tractus olfactorius intermedius.

Do grzbietowo przyśrodkowej strony opuszki przyłączona jest bulbus olfactorius accessorius. Budowa jej przypomina strukturę opuszki węchowej właściwej.

\section{TAFELBESCHREIBUNG}

Tafel XIV.

Abb. 1. Querschnitt des Stratum fibrosum, Stratum glomerulis olfactorii und Stratum moleculare. Präp. 32. Vergr. 7,5 $\times$.

Abb. 2. Querschnitt des Stratum glomerulis olfactorii. Präp. 21. Vergr. $285 \times$.

Abb. 3. Querschnitt der Schicht der mitralen Zellen. Präp. 21. Vergr. $285 \times$.

Abb. 4. Querschnitt des Stratum granulosum internum. Präp. 23. Vergr. $285 \times$.

Abb. 5. Querschnitt längs des Bulbus olfactorius accessorius. Präp. 149. Vergr. $7,5 \times$.

Abb. 6. Querschnitt des Bulbus olfactorius auf der Höhe des anterioren Endes von Neopalium. Präp. 63. Vergr. $7,5 \times$.

Abb. 7. Querschnitt des Bulbus olfactorius accessorius. Präp. 32. Vergr. 7,5×. Abb. 8. Querschnitt des lateralen Teiles des Nebenbulbus olfactorius an seinem posterioren Ende. Präp. 35. Vergr. 7,5 $\times$.

Tafel XV.

Abb. 9. Querschnitt des ventralen Teiles des Bulbus olfactorius an seinem posterioren Ende. Präp. 74. Vergr. 7,5×.

Abb. 10. Zellen des Nucleus olfactorius anterior. Präp. 35. Vergr. $285 \times$.

Abb. 11. Querschnitt des Bulbus olfactorius in seiner halben Länge.

Präp. 25. Vergr. $7,5 \times$.

Abb. 12. Querschnitt des Bulbus olfactorius auf der Höhe des anterioren Ende des Nucleus olfactorius anterior. Präp. 28. Vergr. 7,5×. 


\section{ABKƯRZUNGERKLARUNG}

boa - Bulbus olfactorius accessorius,

apn - Dunkle Fasern im Plexus nervosus,

go - Glomeruli olfactorii,

hpn - Helle Fasern im Plexus nervosus,

kno - Kernzellen des Nucleus olfactorius anterior,

$\mathrm{kz}$ - Körnige Zellen des Stratum granulosum internum,

$\mathrm{mz}$ - Mitrale Zellen,

n - Neopalium,

no - Nucleus olfactorius anterior.

nod - Pars dorsalis Nucleus olfactorius anterior

noel - Pars externa Nucleus olfactorius anterior auf der lateralen Seite des Bulbus olfactorius,

noev - Pars externa Nucleus olfactorius anterior auf der ventralen Seite des Bulbus olfactorius,

nom - Pars medialis Nucleus olfactorius anterior,

non - Ubergang des Nucleus olfactorius anterior ins Neopalium,

pn - Plexus nervosus,

sf - Stratum fibrosum,

sgi - Stratum granulosum internum,

skz - Stratum der körnigen Zellen,

sm - Stratum moleculare,

$\mathrm{smz}$ - Stratum der mitralen Zellen,

toi - Tractus olfactorius intermedius,

tol - Tractus olfactorius lateralis. 


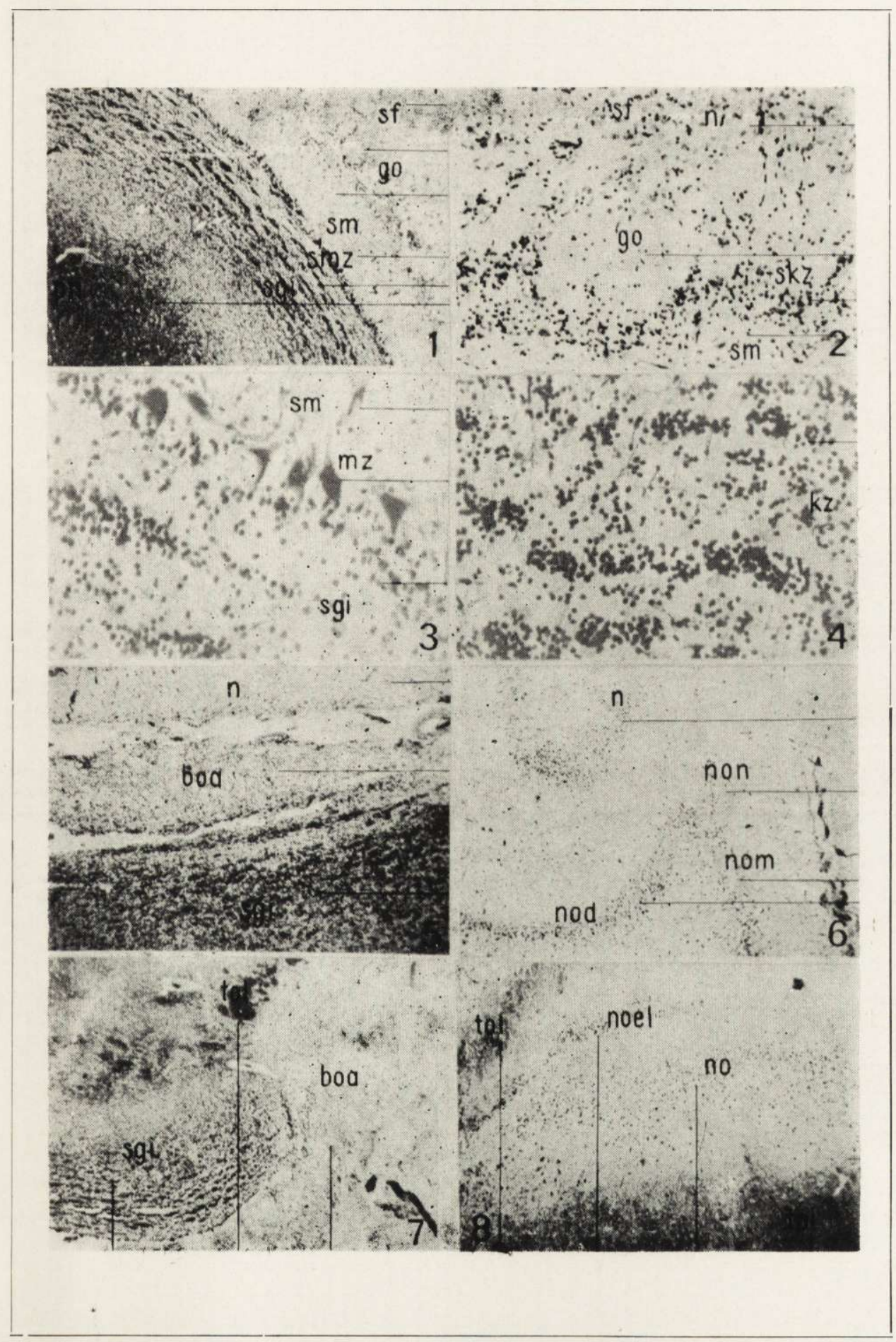

S. Hereć

auctor phot. 


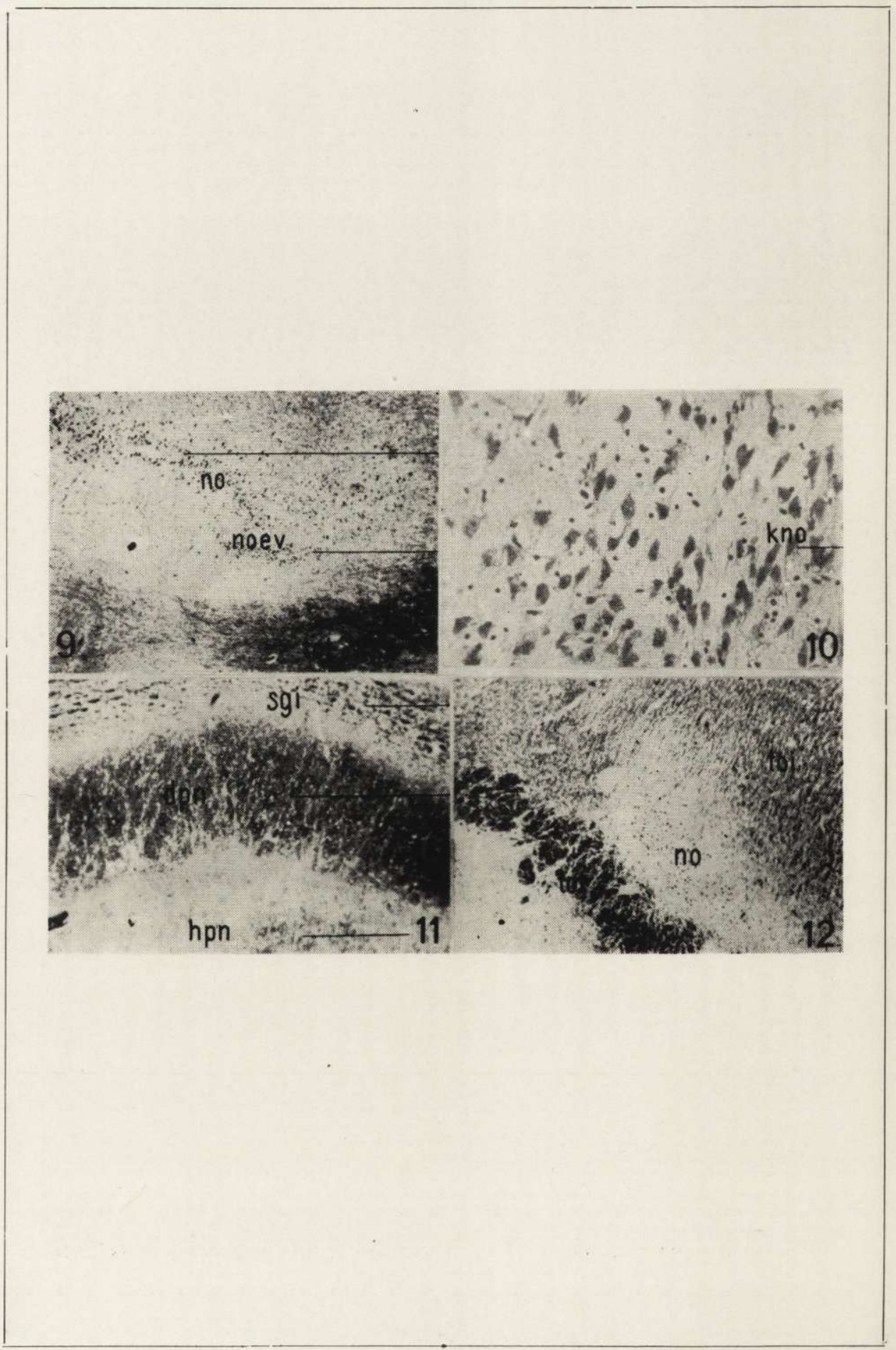

S. Hereć

auctor phot. 